Supporting Information

Direct Organocatalytic Asymmetric Aldol Reactions of $\alpha$-Amino Aldehydes: Expedient Syntheses of Highly Enantiomerically Enriched anti- $\beta$-Hydroxy- $\alpha$-Amino Acids

Rajeswari Thayumanavan, Fujie Tanaka* and Carlos F. Barbas III*

The Skaggs Institute for Chemical Biology and the Departments of Chemistry and Molecular Biology, The Scripps Research Institute, 10550 North Torrey Pines Road, La Jolla, California 92037 (USA)

ftanaka@scripps.edu or carlos@scripps.edu

Phthalimidoacetaldehyde (1). To a stirred mixture of a solution of phthalic anhydride (25 g, $169 \mathrm{mmol})$ in DMF (100 mL) and molecular sieves 4A (5 g), allylamine (15 mL, 200 mmol) was slowly added at rt. The mixture was stirred at $100{ }^{\circ} \mathrm{C}$ for overnight. ${ }^{[\mathrm{S} 1]}$ After cooling to $\mathrm{rt}$, the mixture was filtered through celite and the filtrate was concentrated in vacuo. The residue was dissolved in EtOAc, washed with saturated aqueous $\mathrm{NH}_{4} \mathrm{Cl}$ and with brine, dried over $\mathrm{MgSO}_{4}$, filtered, and concentrated in vacuo. The residue was dissolved in $\mathrm{CH}_{2} \mathrm{Cl}_{2}-\mathrm{MeOH}$ $(9: 1,500 \mathrm{ml})$ and ozone was bubbled through the solution at $-78{ }^{\circ} \mathrm{C}$ for $5 \mathrm{~h}$ until a blue color persisted. The mixture was purged $\mathrm{N}_{2}$ until the blue color disappeared at the same temperature. Dimethylsulfide (90 mL, $1.2 \mathrm{~mol}$ ) was added and the mixture was allowed to warm to $\mathrm{rt}$ and stirred for $16 \mathrm{~h}$ at which point it was concentrated in vacuo. The residue was crystallized from $\mathrm{CH}_{2} \mathrm{Cl}_{2}$ /hexanes to give $\mathbf{1}^{[\mathrm{S} 2]}$ as colorless crystals (22 $\mathrm{g}, 68 \%$ ).

(2S,3S)-2-(1,3-Dioxo-1,3-dihydro-isoindol-2-yl)-3-hydroxy-4-methyl-pentanal (2a)

(Table 1 entry 6). To a mixture of aldehyde 1 (378 mg, $2 \mathrm{mmol})$ and isobutyraldehyde (1.8 mL, 
$20 \mathrm{mmol})$ in NMP $(1 \mathrm{~mL})$ was added L-proline $(69 \mathrm{mg}, 0.6 \mathrm{mmol})$ at $0{ }^{\circ} \mathrm{C}$ and the mixture was stirred at $4{ }^{\circ} \mathrm{C}$. After $36 \mathrm{~h}$, excess isobutyraldehyde was removed in vacuo, and EtOAc and saturated aqueous $\mathrm{NH}_{4} \mathrm{Cl}$ were added to the residue. The organic layer was washed with brine, dried over $\mathrm{MgSO}_{4}$, filtered, concentrated in vacuo, and flash chromatographed (silica gel, EtOAc/hexane $=20: 80)$ to afford 2a $(454 \mathrm{mg}, 87 \%, \mathrm{dr}=>100: 1) .{ }^{1} \mathrm{H}$ NMR $\left(400 \mathrm{MHz}, \mathrm{CDCl}_{3}\right) \delta$ $9.84(\mathrm{~s}, 1 \mathrm{H}, \mathrm{CHO}), 7.91-7.76(\mathrm{~m}, 4 \mathrm{H}), 4.94(\mathrm{~d}, J=5.0 \mathrm{~Hz}, 1 \mathrm{H}, \alpha-\mathrm{H}), 4.15(\mathrm{~m}, 1 \mathrm{H}), 3.67(\mathrm{~d}, J=$ $3.5 \mathrm{~Hz}, 1 \mathrm{H}), 1.98(\mathrm{~m}, 1 \mathrm{H}), 1.11(\mathrm{~d}, J=6.9 \mathrm{~Hz}, 3 \mathrm{H}), 1.06(\mathrm{~d}, J=6.9 \mathrm{~Hz}, 3 \mathrm{H}) ;\left(2 S^{*}, 3 R^{*}\right)$-isomer: 9.71 (s, C

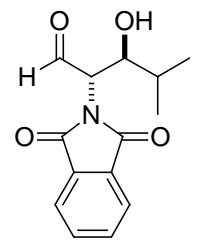

$2 \mathbf{a}$

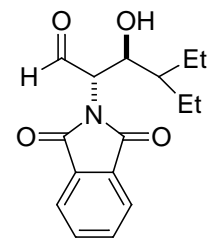

$2 b$

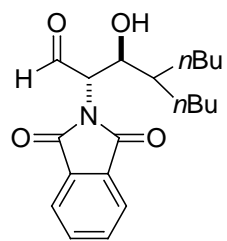

2c

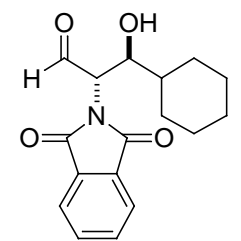

2d

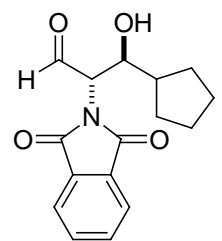

2e

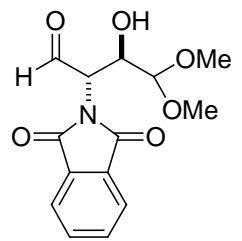

$2 f$

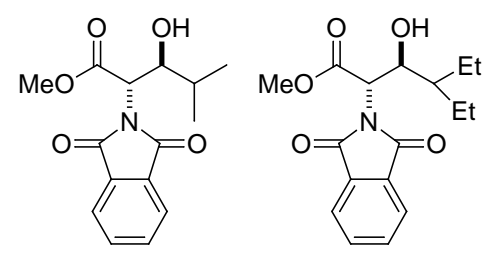

3a

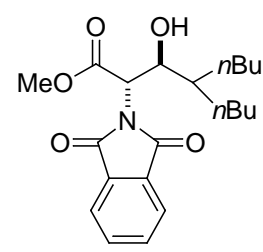

3c

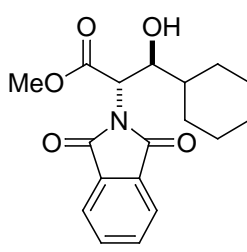

$3 d$

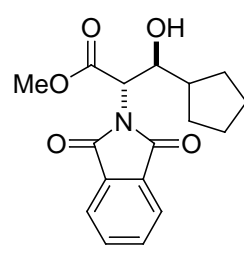

$3 e$

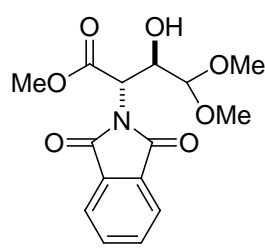

$3 \mathbf{f}$<smiles>CC(C)C(O)[C@H](N)C(=O)O</smiles>

(2S,3S)-2-(1,3-Dioxo-1,3-dihydro-isoindol-2-yl)-3-hydroxy-4-methyl-pentanal $O$ -

benzyl-oxime (8a). Aldehyde 2a was transformed to oxime 8a with $O$-benzylhydroxylamine hydrochloride according to the reported procedure. ${ }^{[\mathrm{S} 3]}{ }^{1} \mathrm{H}$ NMR $\left(400 \mathrm{MHz}, \mathrm{CDCl}_{3}\right) \delta 7.88-7.73$ $(\mathrm{m}, 4 \mathrm{H}), 7.40-7.27(\mathrm{~m}, 6 \mathrm{H}), 5.05-5.02(2 \mathrm{H}), 4.06(\mathrm{~m}, 1 \mathrm{H}), 3.32(\mathrm{~d}, J=3.8 \mathrm{~Hz}, 1 \mathrm{H}), 1.74(\mathrm{~m}$, $1 \mathrm{H}), 1.01(\mathrm{~d}, J=6.7 \mathrm{~Hz}, 3 \mathrm{H}), 0.97(\mathrm{~d}, J=6.7 \mathrm{~Hz}, 3 \mathrm{H})$. HRMS (ESI-TOF): calcd for $\mathrm{C}_{21} \mathrm{H}_{23} \mathrm{~N}_{2} \mathrm{O}_{4}$ 
$\left(\mathrm{MH}^{+}\right)$367.1652, found 367.1655. HPLC (Daicel Chiralcel OD-H, 2-PrOH/hexane = 10:90, flow rate $1.0 \mathrm{~mL} / \mathrm{min}, 254 \mathrm{~nm}):(2 S, 3 S) 19.8 \mathrm{~min},(2 R, 3 R) 38.9 \mathrm{~min}$.

(2S,3S)-2-(1,3-Dioxo-1,3-dihydro-isoindol-2-yl)-3-hydroxy-4-methyl-pentanoic acid methyl ester (3a) (Table 1 entry 7). To a mixture of aldehyde 1 (378 $\mathrm{mg}, 2 \mathrm{mmol}$ ) and isobutyraldehyde $(1.8 \mathrm{~mL}, 20 \mathrm{mmol})$ in NMP $(1 \mathrm{~mL})$ was added L-proline $(69 \mathrm{mg}, 0.6 \mathrm{mmol})$ at 0 ${ }^{\circ} \mathrm{C}$ and the mixture was stirred at $4{ }^{\circ} \mathrm{C}$. After $36 \mathrm{~h}$, excess isobutyraldehyde was removed in vacuo, and EtOAc and saturated aqueous $\mathrm{NH}_{4} \mathrm{Cl}$ were added to the residue. The organic layer was washed with saturated aqueous $\mathrm{NaCl}$, dried over $\mathrm{MgSO}_{4}$, filtered, and concentrated in vacuo. The residue was dissolved in $t \mathrm{BuOH}: \mathrm{H}_{2} \mathrm{O}(5: 1,24 \mathrm{~mL})$ and to this solution was added successively $\mathrm{NaH}_{2} \mathrm{PO}_{4}(480 \mathrm{mg}, 4.0 \mathrm{mmol})$, 2-methyl-2-butene (2M solution in THF, $6.8 \mathrm{~mL}$, $13.6 \mathrm{mmol})$ and $\mathrm{NaClO}_{2}(624 \mathrm{mg}, 6.9 \mathrm{mmol}) .{ }^{[\mathrm{S} 4]}$ The reaction mixture was stirred at $\mathrm{rt}$ for $4 \mathrm{~h}$. The volatile components were removed in vacuo and the residue was extracted with EtOAc. The organic layer was washed with saturated aqueous $\mathrm{NaCl}$, dried over $\mathrm{MgSO}_{4}$, filtered, and concentrated in vасио. The residue was dissolved in toluene $(2 \mathrm{~mL})$ and methanol $(5 \mathrm{~mL})$ and $\mathrm{TMSCHN}_{2}\left(2 \mathrm{M}\right.$ solution in hexane) was added by dropwise at $-20{ }^{\circ} \mathrm{C}$ until the yellow color persisted. The solution was stirred for additional $10 \mathrm{~min}$ and quenched with a drop of acetic acid. The solvents were removed in vacuo and the residue was purified by silica gel flash column chromatography (EtOAc/hexane = 5:95-10:90) to afford 3a (425 mg, 73\% for 3 steps from 1, $\mathrm{dr}=>100: 1,>99.5 \%$ ee $).{ }^{1} \mathrm{H}$ NMR $\left(400 \mathrm{MHz}, \mathrm{CDCl}_{3}\right) \delta 7.92-7.77(\mathrm{~m}, 4 \mathrm{H}), 4.95(\mathrm{~d}, J=$ $6.8 \mathrm{~Hz}, 1 \mathrm{H}), 4.21(\mathrm{~m}, 1 \mathrm{H}), 4.11(\mathrm{~d}, J=2.4 \mathrm{~Hz}, 1 \mathrm{H}), 3.75(\mathrm{~s}, 3 \mathrm{H}), 1.81(\mathrm{~m}, 1 \mathrm{H}), 1.04(\mathrm{~d}, J=6.7$ $\mathrm{Hz}, 3 \mathrm{H}), 0.96(\mathrm{~d}, J=6.7 \mathrm{~Hz}, 3 \mathrm{H}) .{ }^{13} \mathrm{C} \mathrm{NMR}\left(100 \mathrm{MHz}, \mathrm{CDCl}_{3}\right) \delta 170.2,167.7,134.4,131.6$, 123.7, 74.2, 54.6, 52.6, 52.9, 29.6, 19.9, 15.9. IR (neat) 3537, 2960, 1710, 1383, 1249, 1208, 1173, 1086, $716 \mathrm{~cm}^{-1}$. HRMS (ESI-TOF): calcd for $\mathrm{C}_{15} \mathrm{H}_{18} \mathrm{NO}_{5}\left(\mathrm{MH}^{+}\right)$292.1179, found 292.1177. HPLC (Daicel Chiralcel OD-H, 2-PrOH/hexane = 5:95, flow rate $1.0 \mathrm{~mL} / \mathrm{min}, 254$ $\mathrm{nm}):(2 S, 3 S) 10.3 \mathrm{~min},(2 R, 3 R) 13.0 \mathrm{~min} .[\alpha]^{24}+7.1\left(\mathrm{c} 1.0, \mathrm{CHCl}_{3}\right)$.

(2S,3S)-2-(1,3-Dioxo-1,3-dihydro-isoindol-2-yl)-3-hydroxy-4-methyl-pentanoic acid (7). The aldol reaction of $\mathbf{1}$ and isobutyraldehyde and oxidation at Table 1 entry 7 was 
performed using of $1(2.00 \mathrm{~g}, 10.5 \mathrm{mmol})$ and the residue was purified by silica gel flash chromatography $\left(\mathrm{CHCl}_{3} / \mathrm{MeOH}=90: 10\right)$ to afford $7(1.92 \mathrm{~g}, 66 \%) .{ }^{1} \mathrm{H}$ NMR $(400 \mathrm{MHz}$, $\left.\mathrm{CD}_{3} \mathrm{OD}\right) \delta$ 7.86-7.77 (m, 4H), $4.57(\mathrm{~d}, J=9.4 \mathrm{~Hz}, 1 \mathrm{H}), 4.37$ (dd, $\left.J=2.4 \mathrm{~Hz}, 9.4 \mathrm{~Hz}, 1 \mathrm{H}\right), 1.60$ $(\mathrm{m}, 1 \mathrm{H}), 0.97(\mathrm{~d}, J=6.9 \mathrm{~Hz}, 3 \mathrm{H}), 0.85(\mathrm{~d}, J=6.9 \mathrm{~Hz}, 3 \mathrm{H}) .{ }^{13} \mathrm{C} \mathrm{NMR}\left(100 \mathrm{MHz}, \mathrm{CD}_{3} \mathrm{OD}\right) \delta$ 177.1, 169.6, 135.5, 133.2, 124.3, 74.2, 56.1, 30.8, 20.3, 15.0. HRMS (ESI-TOF): calcd for $\mathrm{C}_{14} \mathrm{H}_{16} \mathrm{NO}_{5}\left(\mathrm{MH}^{+}\right)$278.1023, found 278.1025. This acid 7 (5 mg) was transformed to methyl ester $3 \mathbf{a}$ as described above and the ee was determined to be $>99.5 \%$ ee.

(2S,3S)-3-Hydroxyleucine (5). To a stirred solution of 7 (1.92 g, $6.93 \mathrm{mmol})$ in EtOH $(100 \mathrm{~mL})$, anhydrous hydrazine $(35 \mathrm{~mL})$ was added at $\mathrm{rt}$ and the mixture was refluxed for $2 \mathrm{~h}$ until a precipitate of phthalazone was formed. ${ }^{[55]}$ The mixture was cooled to rt and filtered, and the filtrate was concentrated in vacuo. The solid residue was dissolved in water and was washed with $\mathrm{CHCl}_{3}$ (x 5). The aqueous layer was loaded to Dowex 50WX4-100 ion-exchange resin $\left(\mathrm{H}^{+}\right.$ form) and the resin was washed with water $(1 \mathrm{~L})$ then eluted with $2 \mathrm{~N}$ pyridine. ${ }^{[\mathrm{S} 6]}$ The eluted fractions were lyophilized to afford (2S,3S)-hydroxyleucine (5) $(940 \mathrm{mg}, 92 \%$ from 7, 60\% from 1) as a colorless solid. ${ }^{1} \mathrm{H}$ NMR $\left(400 \mathrm{MHz}, \mathrm{D}_{2} \mathrm{O}\right) \delta 3.78(\mathrm{~d}, J=3.2 \mathrm{~Hz}, 1 \mathrm{H}), 3.41(\mathrm{dd}, J=3.2 \mathrm{~Hz}$, $9.0 \mathrm{~Hz}, 1 \mathrm{H}), 1.82(\mathrm{~m}, 1 \mathrm{H}), 0.86(\mathrm{~d}, J=6.4 \mathrm{~Hz}, 3 \mathrm{H}), 0.85(\mathrm{~d}, J=6.4 \mathrm{~Hz}, 3 \mathrm{H})$. HRMS (ESI-TOF): calcd for $\mathrm{C}_{6} \mathrm{H}_{14} \mathrm{NO}_{3}\left(\mathrm{MH}^{+}\right)$148.0968, found 148.0966. $[\alpha]^{25}{ }_{\mathrm{D}}+27.6\left(\mathrm{c} 0.6, \mathrm{H}_{2} \mathrm{O}\right)\left[\right.$ lit. ${ }^{[\mathrm{S} 6]}[\alpha]^{20}{ }_{\mathrm{D}}$ $+20.9\left(\mathrm{c} 1.03, \mathrm{H}_{2} \mathrm{O}\right)$ as $98 \%$ ee; lit. $\left.{ }^{[57]}[\alpha]^{28}{ }_{\mathrm{D}}+37\left(\mathrm{c} 1.0, \mathrm{H}_{2} \mathrm{O}\right)\right]$.

Reactions in Table 2. Aldol reactions in Table 2 were performed using the same procedures as the procedures for Table 1 entry 7, but excess acceptor aldehydes were removed by fast silica gel column chromatography after work-up. The diastereomeric ratio of the aldol products decreased when they were stored or when they were purified by silica gel column chromatography. Attempts to obtain pure aldol products resulted lower yield and lower diasteromeric ratio. Thus, the chromatography was quickly performed to remove excess acceptor aldehyde instead of focusing to purify aldol product 2. Oxidation and esterification were also performed using the same procedures for Table 1 entry 7 as described above. The column chromatography did not completely separate the anti- and syn-isomers of $\mathbf{3}$ each other. 
(2S,3S)-2-(1,3-Dioxo-1,3-dihydro-isoindol-2-yl)-4-ethyl-3-hydroxy-hexanal (2b). ${ }^{1} \mathrm{H}$ NMR (400 MHz, $\left.\mathrm{CDCl}_{3}\right) \delta 9.85(\mathrm{~s}, 1 \mathrm{H}, \mathrm{C} \underline{\mathrm{HO}}), 4.96(\mathrm{~d}, \mathrm{~J}=5.3 \mathrm{~Hz}, 1 \mathrm{H}, \alpha-\mathrm{H})$. For $\left(2 S^{*}, 3 R^{*}\right)$ isomer: $9.70(\mathrm{~s}, 1 \mathrm{H}, \mathrm{C} \underline{\mathrm{HO}}), 5.26(\mathrm{~d}, \alpha-\mathrm{H})$.

(2S,3S)-2-(1,3-Dioxo-1,3-dihydro-isoindol-2-yl)-4-ethyl-3-hydroxy-hexanoic acid methyl ester (3b). ${ }^{1} \mathrm{H}$ NMR $\left(400 \mathrm{MHz}, \mathrm{CDCl}_{3}\right) \delta$ 7.93-7.76 (m, 4H), $4.99(\mathrm{~d}, J=6.8 \mathrm{~Hz}, 1 \mathrm{H}, \alpha-$ H), $4.44(\mathrm{~m}, 1 \mathrm{H}), 3.97(\mathrm{~d}, J=2.4 \mathrm{~Hz}, 1 \mathrm{H}), 3.75(\mathrm{~s}, 3 \mathrm{H}), 1.64-1.18(\mathrm{~m}, 5 \mathrm{H}), 0.88(\mathrm{t}, J=7.5 \mathrm{~Hz}$, $3 \mathrm{H}), 0.82(\mathrm{t}, J=7.5 \mathrm{~Hz}, 3 \mathrm{H}) ;\left(2 S^{*}, 3 R^{*}\right)$-isomer: $5.26(\mathrm{~d}, J=4.1 \mathrm{~Hz}, \alpha-\mathrm{H}) .{ }^{13} \mathrm{C} \mathrm{NMR}(150 \mathrm{MHz}$, $\left.\mathrm{CDCl}_{3}\right) \delta 170.6,167.6,134.4,131.6,123.8,70.9,54.3,52.9,42.5,22.2,20.4,11.6,11.4$. IR (neat) $[(2 S, 3 S)$-isomer:(2S*,3R*)-isomer $=10: 1] 3533,2959,2932,2875,1712,1384,1271$, 1209, 1087, 1017, $717 \mathrm{~cm}^{-1}$. HRMS (ESI-TOF): calcd for $\mathrm{C}_{17} \mathrm{H}_{21} \mathrm{NO}_{5} \mathrm{Na}\left(\mathrm{MNa}^{+}\right) 342.1312$, found 342.1318. HPLC (Daicel Chiralpak AS-H, 2-PrOH/hexane = 5:95, flow rate $1.0 \mathrm{~mL} / \mathrm{min}, 254$ $\mathrm{nm}):(2 S, 3 S) 11.0 \mathrm{~min},(2 R, 3 R) 12.3 \min . \quad[\alpha]_{\mathrm{D}}^{24}-14.2$ (C $\left.1.0, \mathrm{CHCl}_{3}\right)[(2 S, 3 S)-$ isomer: $\left(2 S^{*}, 3 R^{*}\right)$-isomer $=10: 1,(2 S, 3 S)$-isomer $94 \%$ ee $\left.)\right]$.

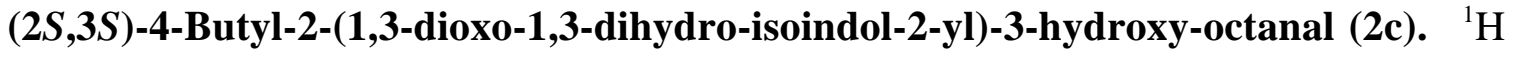
NMR (400 MHz, $\left.\mathrm{CDCl}_{3}\right) \delta 9.85(\mathrm{~s}, 1 \mathrm{H}, \mathrm{CHO}), 4.96(\mathrm{~d}, J=5.6 \mathrm{~Hz}, 1 \mathrm{H}, \alpha-\mathrm{H}) ;\left(2 S^{*}, 3 R^{*}\right)$-isomer: $5.23(\mathrm{~d}, J=5.2 \mathrm{~Hz}, 1 \mathrm{H}, \alpha-\mathrm{H})$.

(2S,3S)-4-Butyl-2-(1,3-dioxo-1,3-dihydro-isoindol-2-yl)-3-hydroxy-octanoic acid methyl ester (3c). ${ }^{1} \mathrm{H}$ NMR $\left(400 \mathrm{MHz}, \mathrm{CDCl}_{3}\right) \delta 7.93-7.76(\mathrm{~m}, 4 \mathrm{H}), 4.98(\mathrm{~d}, J=7.4 \mathrm{~Hz}, 1 \mathrm{H}, \alpha-$ $\mathrm{H}), 4.48(\mathrm{~m}, 1 \mathrm{H}), 3.93(\mathrm{~d}, J=2.6 \mathrm{~Hz}, 1 \mathrm{H}), 3.74(\mathrm{~s}, 3 \mathrm{H}), 1.53-0.82(\mathrm{~m}, 19 \mathrm{H})$; For $\left(2 S^{*}, 3 R^{*}\right)-$ isomer: $5.26(\mathrm{~d}, J=4.4 \mathrm{~Hz}, \alpha-\mathrm{H}) .{ }^{13} \mathrm{C} \mathrm{NMR}\left(100 \mathrm{MHz}, \mathrm{CDCl}_{3}\right) \delta 170.8,167.5,134.4,131.6$, $123.7,71.0,54.2,52.9,39.3,29.8,29.6,29.1,27.8,22.9,14.1$. IR (neat) $[(2 S, 3 S)-$ isomer:(2S*,3R*)-isomer = 7:1] 3541, 2954, 2928, 2859, 1713, 1384, 1262, 1210, 1085, 1018, $718 \mathrm{~cm}^{-1}$. HRMS (ESI-TOF): calcd for $\mathrm{C}_{21} \mathrm{H}_{29} \mathrm{NO}_{5} \mathrm{Na}\left(\mathrm{MNa}^{+}\right) 398.1938$, found 398.1945. HPLC (Daicel Chiralpak AS-H, 2-PrOH/hexane = 5:95, flow rate $1.0 \mathrm{~mL} / \mathrm{min}, 254 \mathrm{~nm}):(2 S, 3 S) 7.1$ $\min ,(2 R, 3 R) 7.8$ min. $[\alpha]^{24}-30.7\left(\mathrm{c} 1.0, \mathrm{CHCl}_{3}\right)[(2 S, 3 S)$-isomer:(2S*,3R*)-isomer = 7:1, $(2 S, 3 S)$-isomer $93 \%$ ee)].

3-Cyclohexyl-2-(1,3-dioxo-1,3-dihydro-isoindol-2-yl)-3-hydroxy-propionaldehyde 


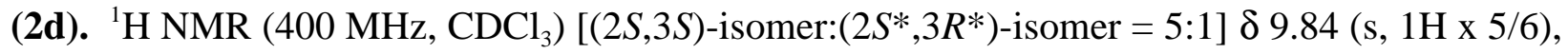
$9.70(\mathrm{~s}, 1 \mathrm{H} \times 1 / 6), 7.97-7.74(\mathrm{~m}, 4 \mathrm{H}), 5.24(\mathrm{~d}, J=4.1 \mathrm{~Hz}, 1 \mathrm{H} \times 1 / 6), 5.00(\mathrm{~d}, J=4.7 \mathrm{~Hz}, 1 \mathrm{H} \times$ 5/6), 4.35 (d, $J=11.3 \mathrm{~Hz}, 1 \mathrm{H} \times 1 / 6), 4.26$ (m, $1 \mathrm{H} \times 1 / 6), 4.11$ (m, 1H x 5/6), 3.96 (brs, $1 \mathrm{H} \times 5 / 6)$, 2.25-0.90 (m, 11H).

\section{3-Cyclohexyl-2-(1,3-dioxo-1,3-dihydro-isoindol-2-yl)-3-hydroxy-propionic acid} methyl ester (3d). ${ }^{1} \mathrm{H} \mathrm{NMR}\left(400 \mathrm{MHz}, \mathrm{CDCl}_{3}\right)\left[(2 S, 3 S)\right.$-isomer: $\left(2 S^{*}, 3 R^{*}\right)$-isomer $\left.=5: 1\right] \delta 7.93$ $7.77(\mathrm{~m}, 4 \mathrm{H}), 5.26(\mathrm{~d}, J=4.1 \mathrm{~Hz}, 1 \mathrm{H}$ x 1/6), $5.01(\mathrm{~d}, J=5.9 \mathrm{~Hz}, 1 \mathrm{H}$ x 5/6), $4.42(\mathrm{~d}, J=11.4 \mathrm{~Hz}$, $1 \mathrm{H} \times 1 / 6), 4.17-4.01(\mathrm{~m}, 2 \mathrm{H}), 3.80(\mathrm{~s}, 1 \mathrm{H} \times 1 / 6) .3 .75(\mathrm{~s}, 3 \mathrm{H} \times 5 / 6), 2.21(\mathrm{~m}, 1 \mathrm{H} \times 1 / 6), 1.89(\mathrm{~m}$,

$1 \mathrm{H} \times 5 / 6), 1.79-0.90(\mathrm{~m}, 10 \mathrm{H}) .{ }^{13} \mathrm{C} \mathrm{NMR}\left(125 \mathrm{MHz}, \mathrm{CDCl}_{3}\right)[(2 S, 3 S)$-isomer $] \delta 170.0,167.8$, $134.4,123.7,74.3,54.4,52.8,39.6,30.0,26.7,26.2,25.9 . \quad$ IR (neat) $[(2 S, 3 S)$-isomer:(2S*,3R*)isomer $=5: 1] 3468,2924,2851,1708,1384,1254,1210,1085,1068,1018,719 \mathrm{~cm}^{-1}$. HRMS (ESI-TOF): calcd for $\mathrm{C}_{18} \mathrm{H}_{21} \mathrm{NO}_{5} \mathrm{Na}\left(\mathrm{MNa}^{+}\right)$354.1312, found 354.1323. HPLC (Daicel Chiralcel OD-H, 2-PrOH/hexane = 5:95, flow rate $1.0 \mathrm{~mL} / \mathrm{min}, 254 \mathrm{~nm}):(2 S, 3 S) 9.7 \mathrm{~min},(2 R, 3 R) 11.1$ min. $[\alpha]_{\mathrm{D}}^{24}-13.7\left(\mathrm{c} 0.75, \mathrm{CHCl}_{3}\right)\left[(2 S, 3 S)\right.$-isomer: $\left(2 S^{*}, 3 R^{*}\right)$-isomer $=5: 1,(2 S, 3 S)$-isomer $=$ $98 \%$ ee)].

\section{3-Cyclopentyl-2-(1,3-dioxo-1,3-dihydro-isoindol-2-yl)-3-hydroxy-propionaldehyde}

(2e). ${ }^{1} \mathrm{H}$ NMR (400 MHz, $\left.\mathrm{CDCl}_{3}\right)\left[(2 S, 3 S)\right.$-isomer: $\left(2 S^{*}, 3 R^{*}\right)$-isomer = 3:1] $\delta 9.85(\mathrm{~s}, 1 \mathrm{H} \mathrm{x} 3 / 4)$, $9.71(\mathrm{~s}, 1 \mathrm{H} \times 1 / 4), 7.93-7.70(\mathrm{~m}, 4 \mathrm{H}), 5.16(\mathrm{~d}, J=2.1 \mathrm{~Hz}, 1 \mathrm{H} \times 1 / 4), 4.96(\mathrm{~d}, J=3.0 \mathrm{~Hz}, 1 \mathrm{H} \mathrm{x}$ 3/4), 4.35-4.33 (2H x 1/4), 4.12-4.09 (2H x 3/4), 2.40-1.20 (m, 9H).

(2S,3S)-3-Cyclopentyl-2-(1,3-dioxo-1,3-dihydro-isoindol-2-yl)-3-hydroxy-propionic acid methyl ester (3e). ${ }^{1} \mathrm{H}$ NMR $\left(400 \mathrm{MHz} \mathrm{CDCl}_{3}\right) \delta$ 7.92-7.76 (m, 4H), $4.94(\mathrm{~d}, J=4.7 \mathrm{~Hz}$, $1 \mathrm{H}, \alpha-\underline{\mathrm{H}}), 4.32(\mathrm{~d}, J=2.9 \mathrm{~Hz}, 1 \mathrm{H}), 4.13(\mathrm{~m}, 1 \mathrm{H}), 3.76(\mathrm{~s}, 3 \mathrm{H}), 2.23(\mathrm{~m}, 1 \mathrm{H}), 1.81-1.33(\mathrm{~m}, 8 \mathrm{H})$; $\left(2 S^{*}, 3 R^{*}\right)$-isomer: $5.19(\mathrm{~d}, J=4.1 \mathrm{~Hz}, \alpha-\mathrm{H}) .{ }^{13} \mathrm{C} \mathrm{NMR}\left(100 \mathrm{MHz}, \mathrm{CDCl}_{3}\right) \quad \delta 169.5,167.9$, $134.5,131.6,123.8,74.2,56.5,52.8,42.1,29.3,28.0,25.8$. IR (neat) $[(2 S, 3 S)-$ isomer:(2S*,3R*)-isomer = 16:1] 3469, 2951, 2867, 1708, 1384, 1250, 1208, 1085, 1019, 718. HRMS (ESI-TOF): calcd for $\mathrm{C}_{17} \mathrm{H}_{19} \mathrm{NO}_{5} \mathrm{Na}\left(\mathrm{MNa}^{+}\right)$340.1155, found 340.1149. [ $\left.\alpha\right]_{\mathrm{D}}^{24}+26.2(\mathrm{c}$ 1.0, $\left.\mathrm{CHCl}_{3}\right)\left[(2 S, 3 S)\right.$-isomer: $\left(2 S^{*}, 3 R^{*}\right)$-isomer $\left.=16: 1\right]$. 


\section{3-Cyclopentyl-2-(1,3-dioxo-1,3-dihydro-isoindol-2-yl)-3-hydroxy-propionaldehyde}

O-benzyl-oxime (8e). ${ }^{1} \mathrm{H}$ NMR (400 MHz, $\mathrm{CDCl}_{3}$ ) $\delta$ 7.89-7.74 (m, 4H), 7.40-7.26 (6H), 5.07 (s, 2H), $3.99(\mathrm{~m}, 1 \mathrm{H}), 3.74(\mathrm{~d}, J=2.9 \mathrm{~Hz}, 1 \mathrm{H}), 1.98(\mathrm{~m}, 1 \mathrm{H}), 1.84-1.28(\mathrm{~m}, 8 \mathrm{H})$. HPLC (Daicel Chiralpak AD, 2-PrOH/hexane = 10:90, flow rate $1.0 \mathrm{~mL} / \mathrm{min}, 254 \mathrm{~nm}):(2 S, 3 S) 48.3 \mathrm{~min}$, $(2 R, 3 R) 26.3 \mathrm{~min}$.

\section{2-(1,3-Dioxo-1,3-dihydro-isoindol-2-yl)-3-hydroxy-4,4-dimethoxy-butyraldehyde}

(2f). ${ }^{1} \mathrm{H}$ NMR $\left(400 \mathrm{MHz}, \mathrm{CDCl}_{3}\right)\left[\right.$ anti-(2S,3R)-isomer:syn-( $\left.2 S^{*}, 3 S^{*}\right)$-isomer $\left.=5: 1\right] \delta 9.88(\mathrm{~s}$, $1 \mathrm{H} \times 5 / 6), 9.73$ (s, $1 \mathrm{H} \times 1 / 6), 7.95-7.70(\mathrm{~m}, 4 \mathrm{H}), 5.22$ (d, $J=4.4 \mathrm{~Hz}, 1 \mathrm{H} \times 5 / 6), 5.19$ (d, $J=5.0$ $\mathrm{Hz}, 1 \mathrm{H} \times 1 / 6), 4.63$ (m, $1 \mathrm{H} \times 1 / 6), 4.58(\mathrm{~d}, J=5.9 \mathrm{~Hz}, 1 \mathrm{H} \times 5 / 6), 4.37-4.25(\mathrm{~m}, 1 \mathrm{H}), 3.52(\mathrm{~s}, 3 \mathrm{H}$ x 5/6), 3.48 (s, $3 \mathrm{H} \times 5 / 6), 3.45(\mathrm{~s}, 3 \mathrm{H} \mathrm{x} \mathrm{1/6),} 3.41$ (s, $3 \mathrm{H} \mathrm{x} \mathrm{1/6).}$

2-(1,3-Dioxo-1,3-dihydro-isoindol-2-yl)-3-hydroxy-4,4-dimethoxy-butyric acid methyl ester (3f). ${ }^{1} \mathrm{H}$ NMR $\left(400 \mathrm{MHz}, \mathrm{CDCl}_{3}\right)\left[\right.$ anti-(2S,3R)-isomer:syn-( $\left.2 S^{*}, 3 S^{*}\right)$-isomer $=$ 1:1] $\delta 7.93-7.75(\mathrm{~m}, 4 \mathrm{H}), 5.33$ (d, $J=4.4 \mathrm{~Hz}, 1 \mathrm{H} \times 1 / 2), 5.26$ (d, $J=3.5 \mathrm{~Hz}, 1 \mathrm{H} \times 1 / 2), 4 / 69$ (d, $J$ $=6.8 \mathrm{~Hz}, 1 \mathrm{H} \mathrm{x} \mathrm{1/2),} 4.54(\mathrm{~m}, 1 \mathrm{H} \times 1 / 2), 4.45-4.25(\mathrm{~m}, 2 \mathrm{H}), 3.79(\mathrm{~d}, J=4.7 \mathrm{~Hz}, 3 \mathrm{H}), 3.42(\mathrm{~d}, J=$ $4.7 \mathrm{~Hz}, 3 \mathrm{H}), 3.41(\mathrm{~s}, 3 \mathrm{H} \times 1 / 2), 3.36(\mathrm{~s}, 3 \mathrm{H} \times 1 / 2) .{ }^{13} \mathrm{C} \mathrm{NMR}\left(125 \mathrm{MHz}, \mathrm{CDCl}_{3}\right)[$ antiisomer:syn-isomer $=1: 1] \delta 168.6,168.5,168.2,168.0,134.3,131.7,123.8,123.7,104.3,103.9$, 104.3, 103.9, 71.1, 70.3, 56.1, 55.3, 54.7, 54.6, 54.4, 53.9, 52.9, 52.8. IR (neat) [anti-isomer:synisomer $=1: 1] 3436,2953,2835,1746,1705,1387,1264,1193,1114,1063,970,718$. HRMS (ESI-TOF): calcd for $\mathrm{C}_{15} \mathrm{H}_{17} \mathrm{NO}_{7} \mathrm{Na}\left(\mathrm{MNa}^{+}\right)$346.0897, found 346.0899. HPLC (Daicel Chiralcel OD-H, 2-PrOH/hexane = 10:90, flow rate $1.0 \mathrm{~mL} / \mathrm{min}, 254 \mathrm{~nm})$ : anti-isomers, $(2 S, 3 R) 44.7 \mathrm{~min}$, $(2 R, 3 S) 72.6 \mathrm{~min}$; syn-isomer, $36.8 \mathrm{~min}, 54.1 \mathrm{~min} .[\alpha]^{24}+19.8\left(\mathrm{c} 1.0, \mathrm{CHCl}_{3}\right)$ [anti-isomer:synisomer $=1: 1$, anti-isomer $86 \%$ ee, syn-isomer $68 \%$ ee].

4-(1,3-Dioxo-1,3-dihydro-isoindol-2-yl)-3-hydroxy-2-isopropyl-butyraldehyde (6a). ${ }^{1} \mathrm{H} \mathrm{NMR}\left(400 \mathrm{MHz}, \mathrm{CDCl}_{3}\right)$ (anti:syn = 3:1) $\delta 9.90(\mathrm{~d}, J=2.3 \mathrm{~Hz}, 1 \mathrm{H} \mathrm{x} 1 / 4), 9.87(\mathrm{~d}, J=3.0 \mathrm{~Hz}$, $1 \mathrm{H} \times 3 / 3), 7.89-7.70(\mathrm{~m}, 4 \mathrm{H}), 4.31(\mathrm{~m}, 1 \mathrm{H} \times 1 / 4), 4.26(\mathrm{~m}, 1 \mathrm{H} \times 3 / 4), 3.91-3.81(2 \mathrm{H}), 3.27$ (brs, $1 \mathrm{H} \mathrm{x} \mathrm{3/4),} 2.95$ (brs, $1 \mathrm{H} \mathrm{x} \mathrm{1/4),} \mathrm{2.53-2.10} \mathrm{(m,} \mathrm{2H),} 1.16$ (d, $J=6.8 \mathrm{~Hz}, 3 \mathrm{H} \times 1 / 4), 1.10$ (d, $J=6.8$ $\mathrm{Hz}, 3 \mathrm{H} \times 3 / 4), 1.06(\mathrm{~d}, J=6.8 \mathrm{~Hz}, 3 \mathrm{H} \times 1 / 4) .1 .04(\mathrm{~d}, J=6.8 \mathrm{~Hz}, 3 \mathrm{H} \times 1 / 4) .{ }^{13} \mathrm{C}$ NMR $(125$ 
$\mathrm{MHz}, \mathrm{CDCl}_{3}$ ) (anti-isomer) $\delta$ 206.0, 168.7, 134.1, 131.7, 123.4, 69.0, 60.6, 43.0, 27.0, 20.8, 19.7. HPLC (Daicel Chiralcel OD-H, 2-PrOH/hexane $=10: 90$, flow rate $1.0 \mathrm{~mL} / \mathrm{min}, 254 \mathrm{~nm}$ ): anti-isomer, major $17.0 \mathrm{~min}$, minor $53.1 \mathrm{~min}$.

2-[2-(1,3-Dioxo-1,3-dihydro-isoindol-2-yl)-1-hydroxy-ethyl]-hexanal (6b). ${ }^{1} \mathrm{H}$ NMR $\left(400 \mathrm{MHz}, \mathrm{CDCl}_{3}\right)($ anti:syn $=3: 1) \delta 9.83(\mathrm{~d}, J=1.4 \mathrm{~Hz}, 1 \mathrm{H} \mathrm{x} \mathrm{1/4}), 9.77(\mathrm{~d}, J=2.9 \mathrm{~Hz}, 1 \mathrm{H} \mathrm{x}$ 3/4), 7.90-7.75 (m, 4H), 4.26 (m, 1H x 1/4), 4.12 (m, 1H x 3/4), 3.96-3.86 (m, 2H), $3.10(\mathrm{~m}, 1 \mathrm{H}$ x 3/4), $2.82(\mathrm{~m}, 1 \mathrm{H}$ x 1/4), 2.57-0.90 (m, 9H). HPLC (Daicel Chiralcel OD-H, 2-PrOH/hexane = 10:90, flow rate $1.0 \mathrm{~mL} / \mathrm{min}, 254 \mathrm{~nm}$ ): anti-isomer, major $16.1 \mathrm{~min}$, minor $36.3 \mathrm{~min}$.

\section{References}

(S1) M. Palucki, D. H. Hughes, N. Yasuda, C. Yang, P. J. Reider, Tetrahedron Lett. 2001, 42,6811 .

(S2) Aldehyde $\mathbf{1}$ is commercially available in some countries.

(S3) W. Notz, F. Tanaka, S. Watanabe, N. S. Chowdari, J. M. Turner, R. Thayumanavan, C. F. Barbas, III, J. Org. Chem. 2003, 68, 9624.

(S4) Y. Deng, R. G. Salomon, J. Org. Chem. 2000, 65, 6660.

(S5) J. Mulzer, A. Angermann, S. Schubert, C. Seiz, J. Org. Chem. 1986, 51, 5294.

(S6) K. Makino, N. Okamoto, O. Hara, Y. Hamada, Tetrahedron: Asymmetry 2001, 12, 1757.

(S7) Nagamitsu, T. Sunazuka, H. Tanaka, S. Omura, P. A. Sprengeler, A. B. Smith, III, J. Am. Chem. Soc. 1996, 118, 3584. 
Compound 3a, ${ }^{1} \mathrm{H}$ NMR (400 MHz, $\mathrm{CDCl}_{3}$ )
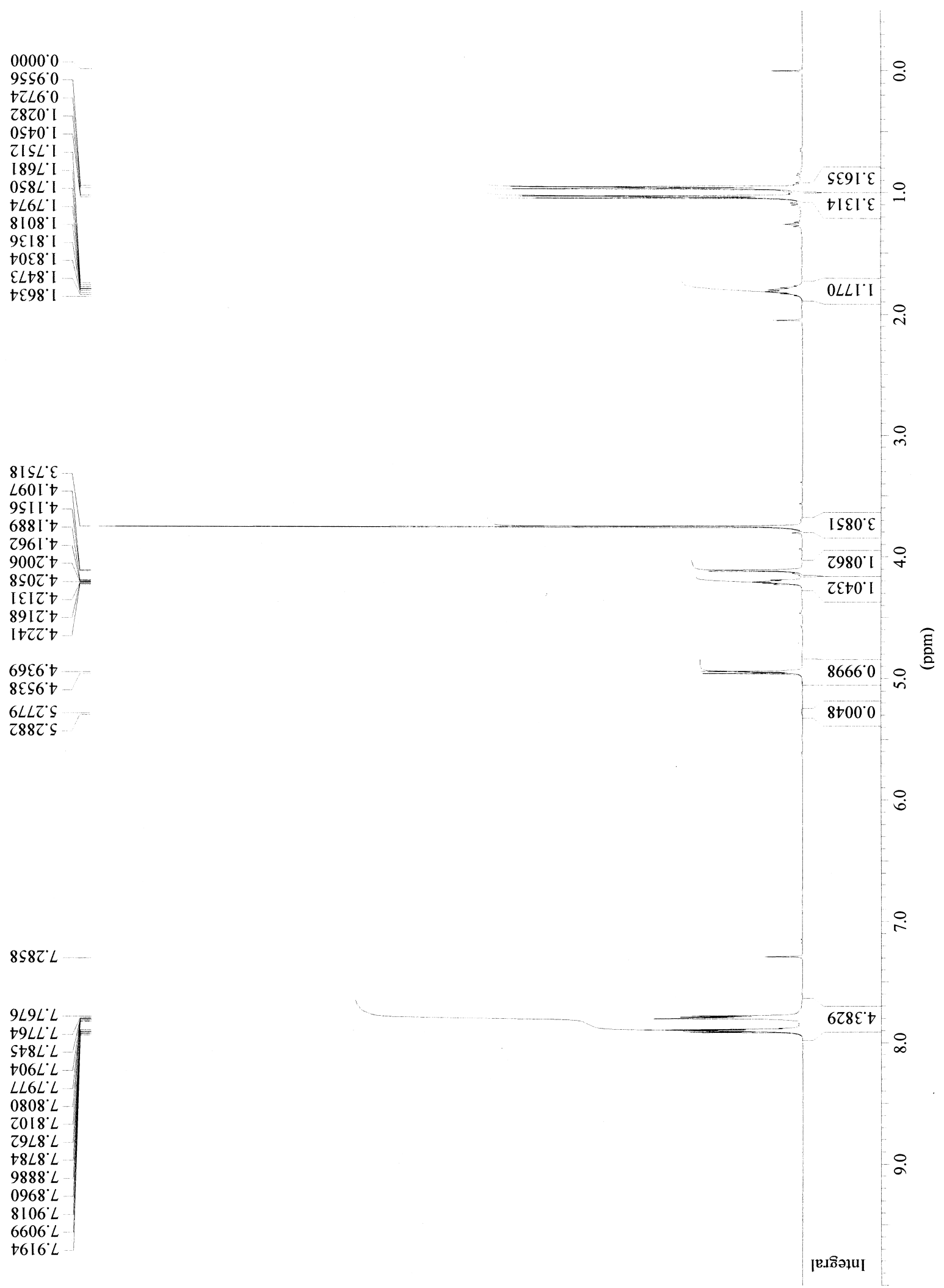
Compound 3a, ${ }^{13} \mathrm{C}$ NMR $\left(100 \mathrm{MHz}, \mathrm{CDCl}_{3}\right)$

$\varepsilon 288^{\circ} \varsigma \mathrm{I}$
$0988^{\circ} 6 \mathrm{I}$
$I Z 6 \varsigma^{\circ} 6 Z$

$t I \angle 8^{\circ} Z S^{\circ}$
I $S S^{\circ} \mathrm{S}$

$0+9 I^{\circ} \mathrm{t} L$

SI89.9L

$0000^{\circ} L L$

SIIELL

$\angle 0 t L^{\circ} \varepsilon Z I$

Z995. $I \varepsilon$

9Ltt๋

S099 $\angle 9 \mathrm{I}$

$\angle 8 \& Z^{\circ} 0 \angle \mathrm{I}$

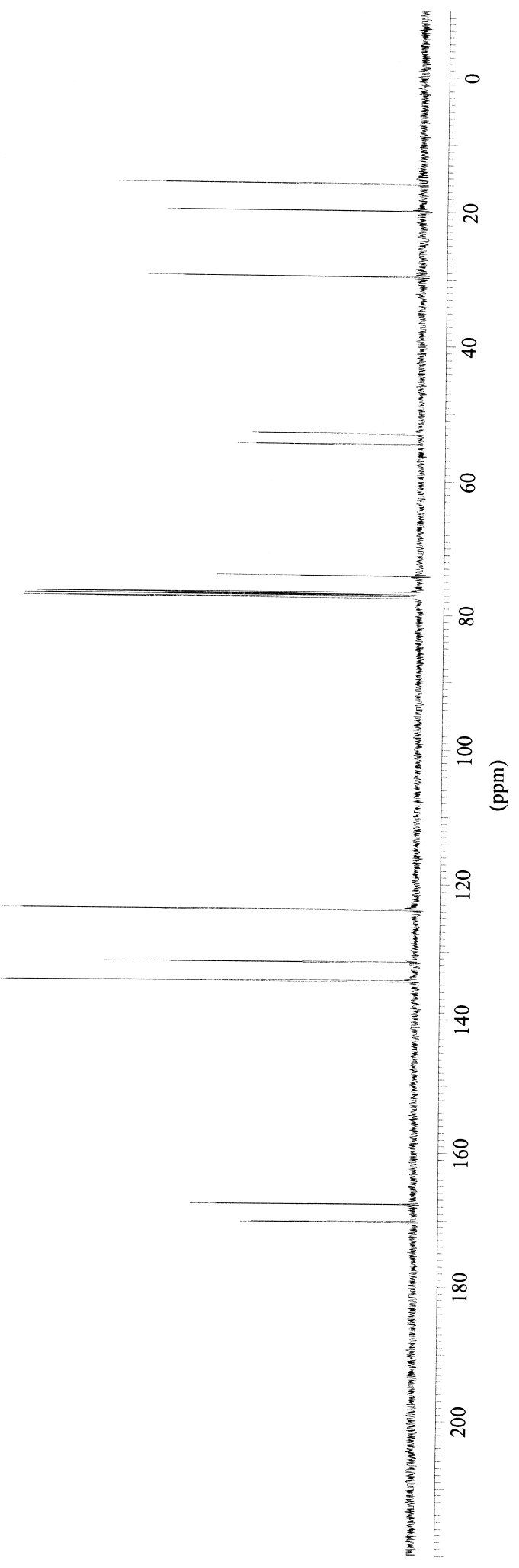

S10 


\section{Compound 7, ${ }^{1} \mathrm{H}$ NMR (400 MHz, $\mathrm{CD}_{3} \mathrm{OD}$ )}

$8 \mathrm{It8} 8^{\circ} 0$

$\angle 8 S 8^{\circ} 0$

$9096^{\circ} 0$

$28 \angle 6^{\circ} 0$

$\angle 29 S^{\circ} \mid$

989S' I

$88 \angle S^{\circ} \mathrm{I}$

$\angle 78 S^{\circ} \mathrm{I}$

t965' I

$\varepsilon 209^{\circ} \mathrm{I}$

EEI9'I

26I9. I

20E9.1

$616 \tau^{\circ} \mathcal{E}$

$\varepsilon 96 \tau^{\circ} \mathcal{\varepsilon}$

$000 \mathcal{E}^{\circ} \mathcal{E}$

${ }^{\circ} 0 \mathcal{E}^{\circ} \mathcal{E}$

I $80 \mathcal{E}^{\circ} \mathcal{E}$

$68 \mathcal{E} \mathcal{E}^{\circ} \mathcal{E}$

09SE'

6I9E'

S6LE'

$\rightarrow S 8 \varepsilon^{\circ} t$

EESS

89 LS" $^{\circ}$

$\angle 0 Z 6^{\circ} t$

$\varepsilon \angle 8 L^{\circ} L$

${ } \subseteq 6 L^{\circ} L$

$0 Z 08^{\circ} \mathrm{L}$

$\mathcal{E} 608^{\circ} \mathrm{L}$

$6818^{\circ} \mathrm{L}$

$7828^{\circ} \mathrm{L}$

$\angle 8 E 8^{\circ} \mathrm{L}$

ESt8. $\mathrm{L}$

$6 I S 8^{\circ} \mathrm{L}$

$66 \mathrm{~S}^{\circ} \mathrm{L}$

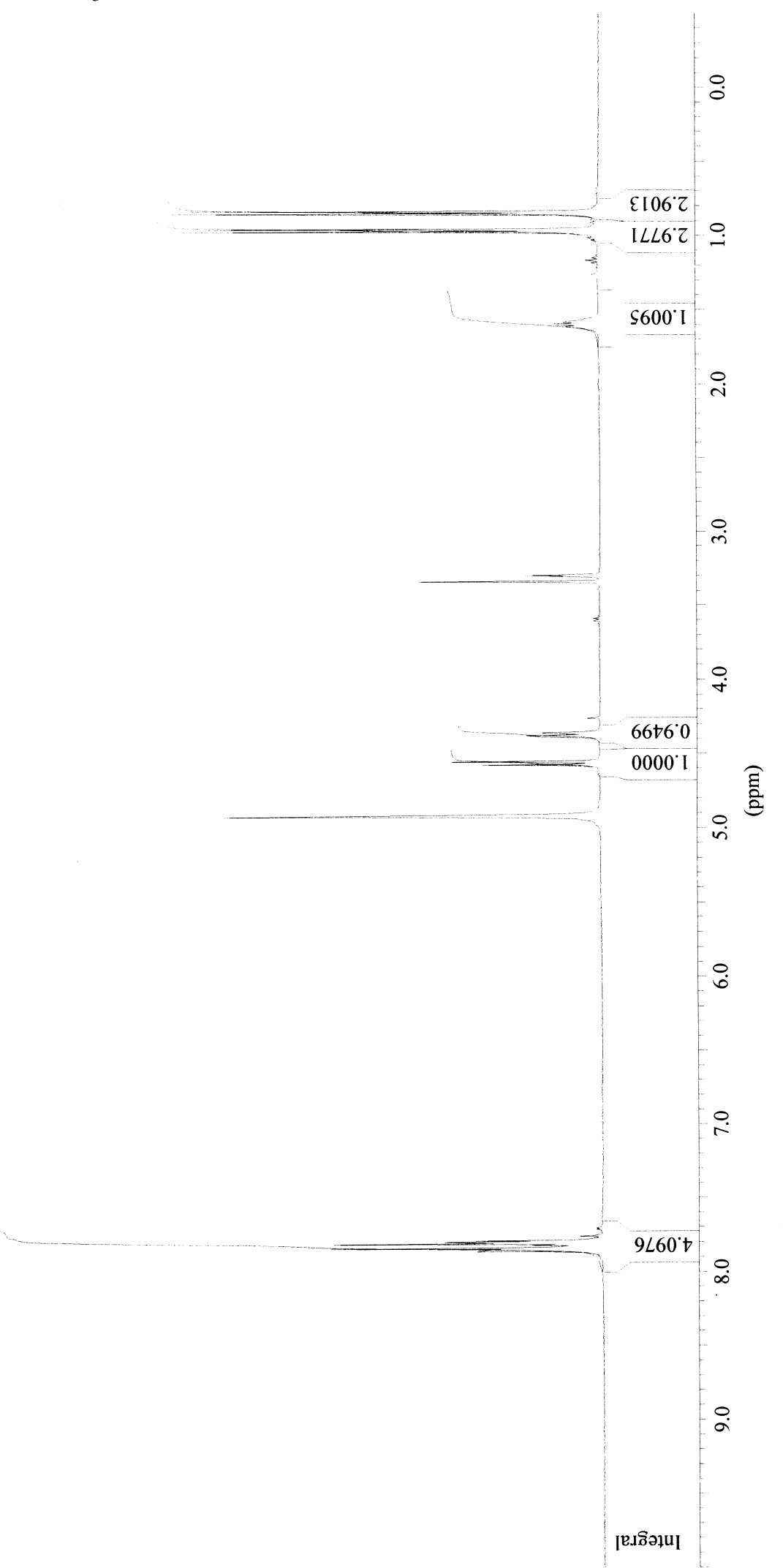




\section{Compound 7, ${ }^{13} \mathrm{C}$ NMR (100 MHz, $\left.\mathrm{CD}_{3} \mathrm{OD}\right)$}

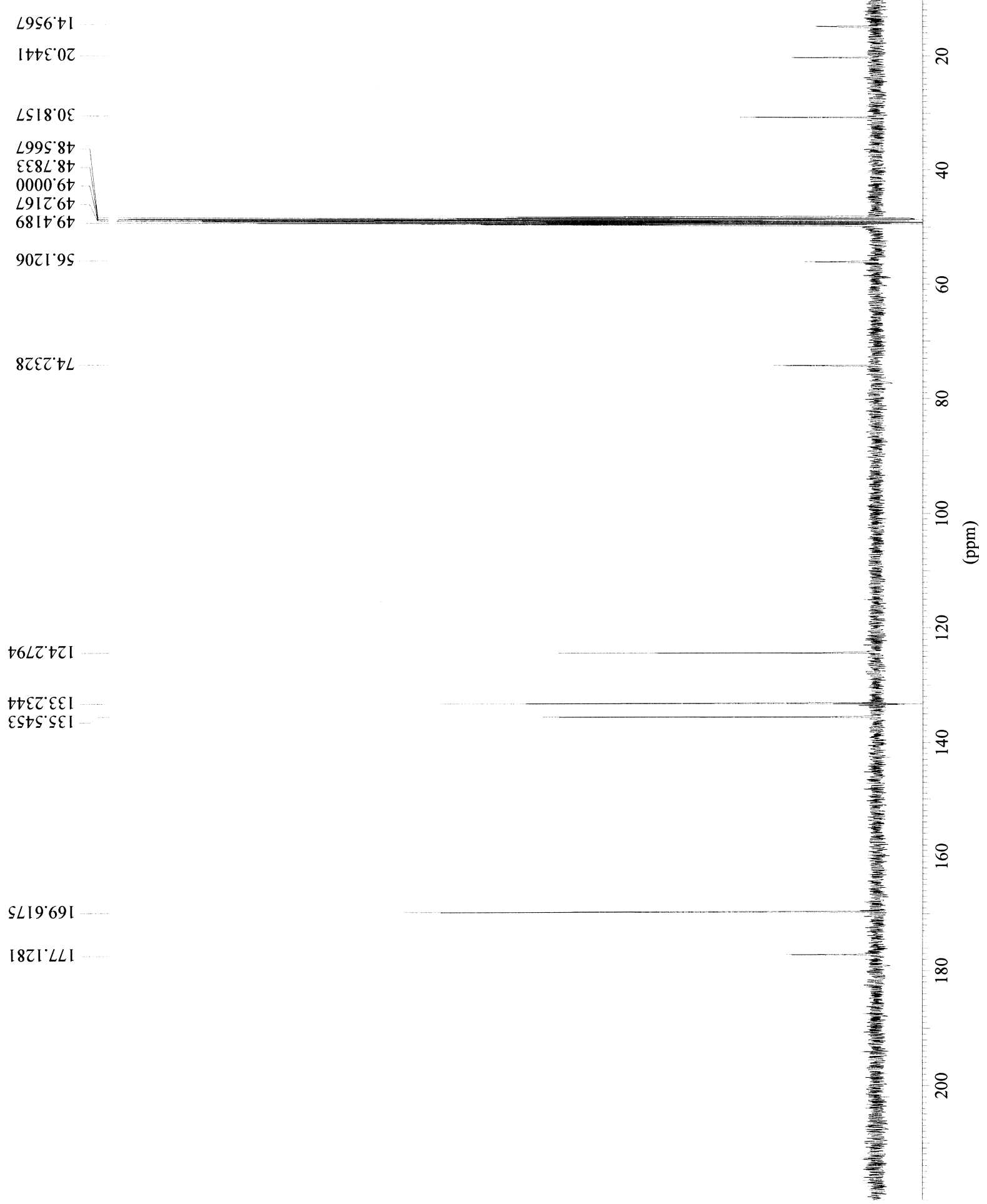


Compound 3b, ${ }^{1} \mathrm{H}$ NMR (400 MHz, $\mathrm{CDCl}_{3}$ )

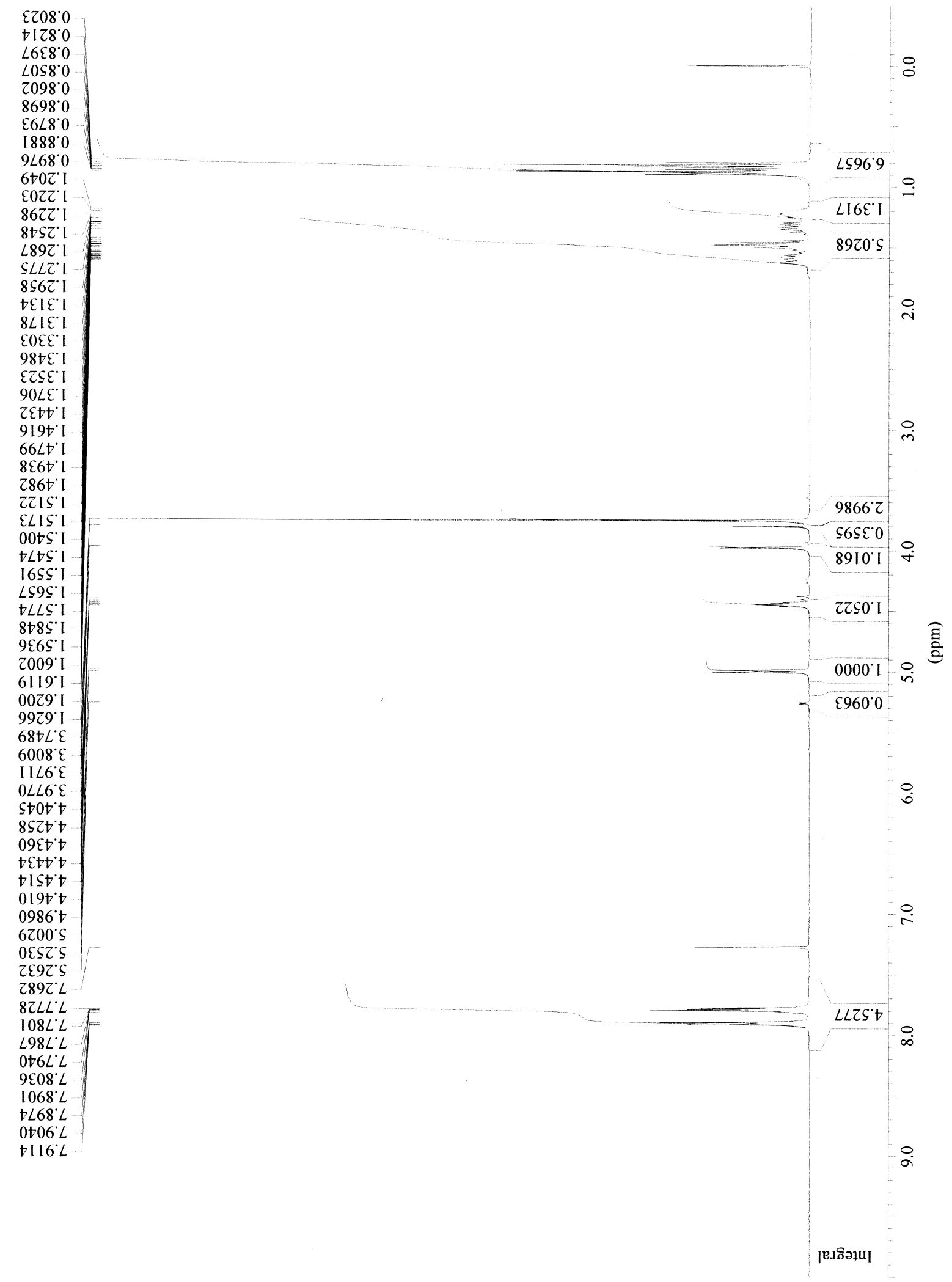


Compound 3b, ${ }^{13} \mathrm{C}$ NMR $\left(150 \mathrm{MHz}, \mathrm{CDCl}_{3}\right)$

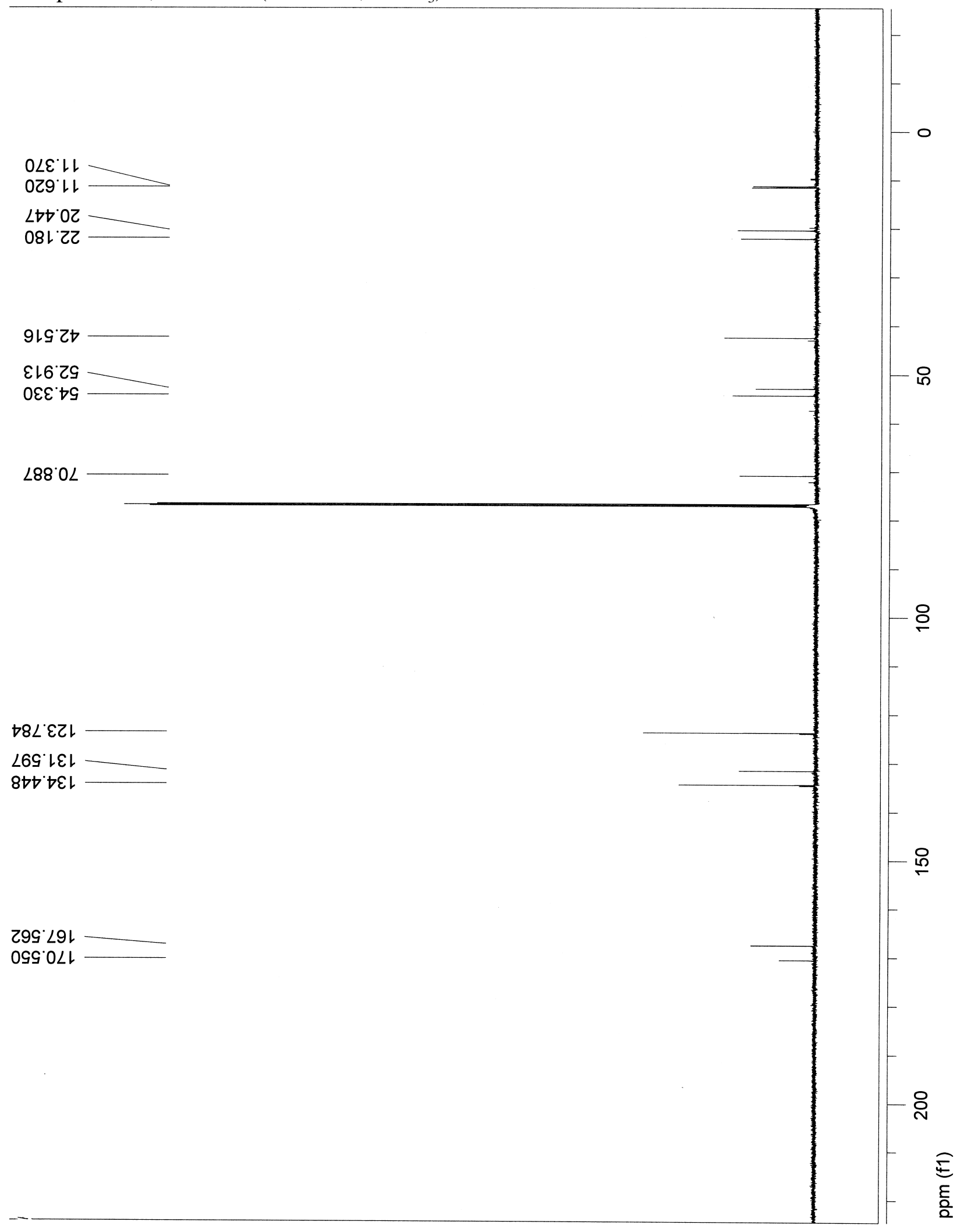

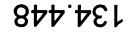

299 $\angle 9$ L

OSG. $0<1$ 
Compound 3c, ${ }^{1} \mathrm{H}$ NMR $\left(400 \mathrm{MHz}, \mathrm{CDCl}_{3}\right.$ )

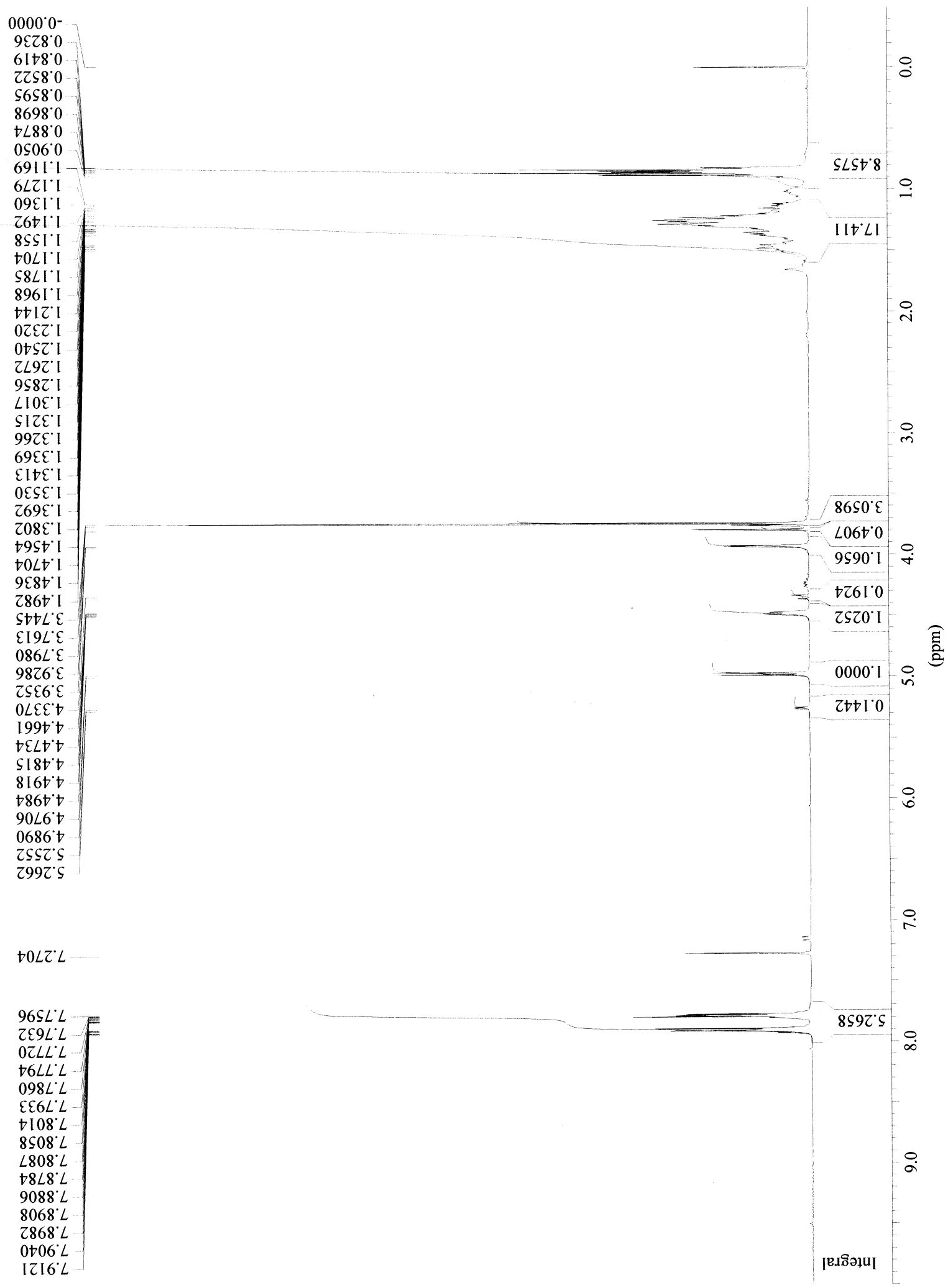


Compound 3c, ${ }^{13} \mathrm{C}$ NMR $\left(100 \mathrm{MHz}, \mathrm{CDCl}_{3}\right)$

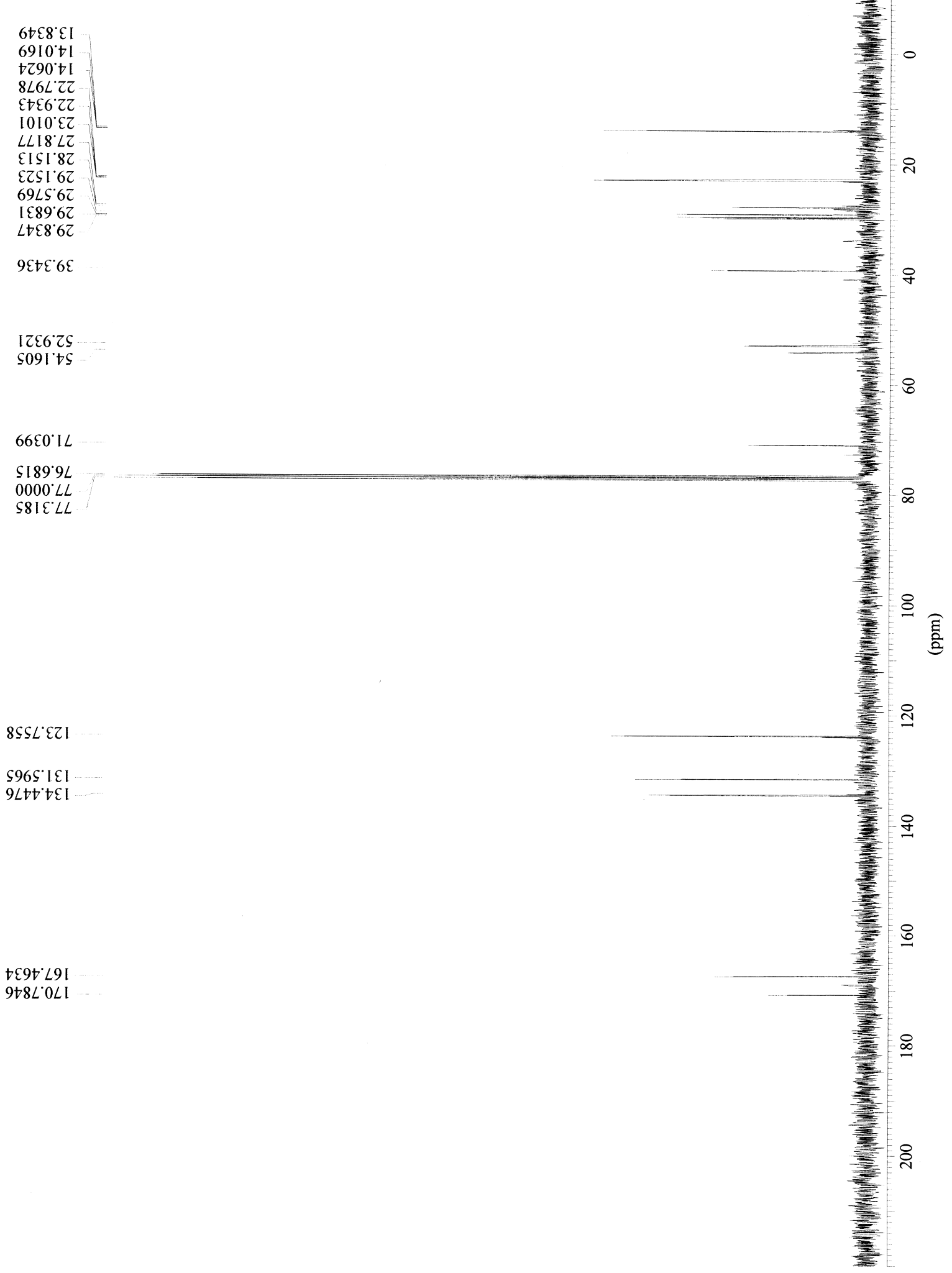


Compound 3d, anti:syn $=5: 1,{ }^{1} \mathrm{H}$ NMR $\left(400 \mathrm{MHz}, \mathrm{CDCl}_{3}\right)$

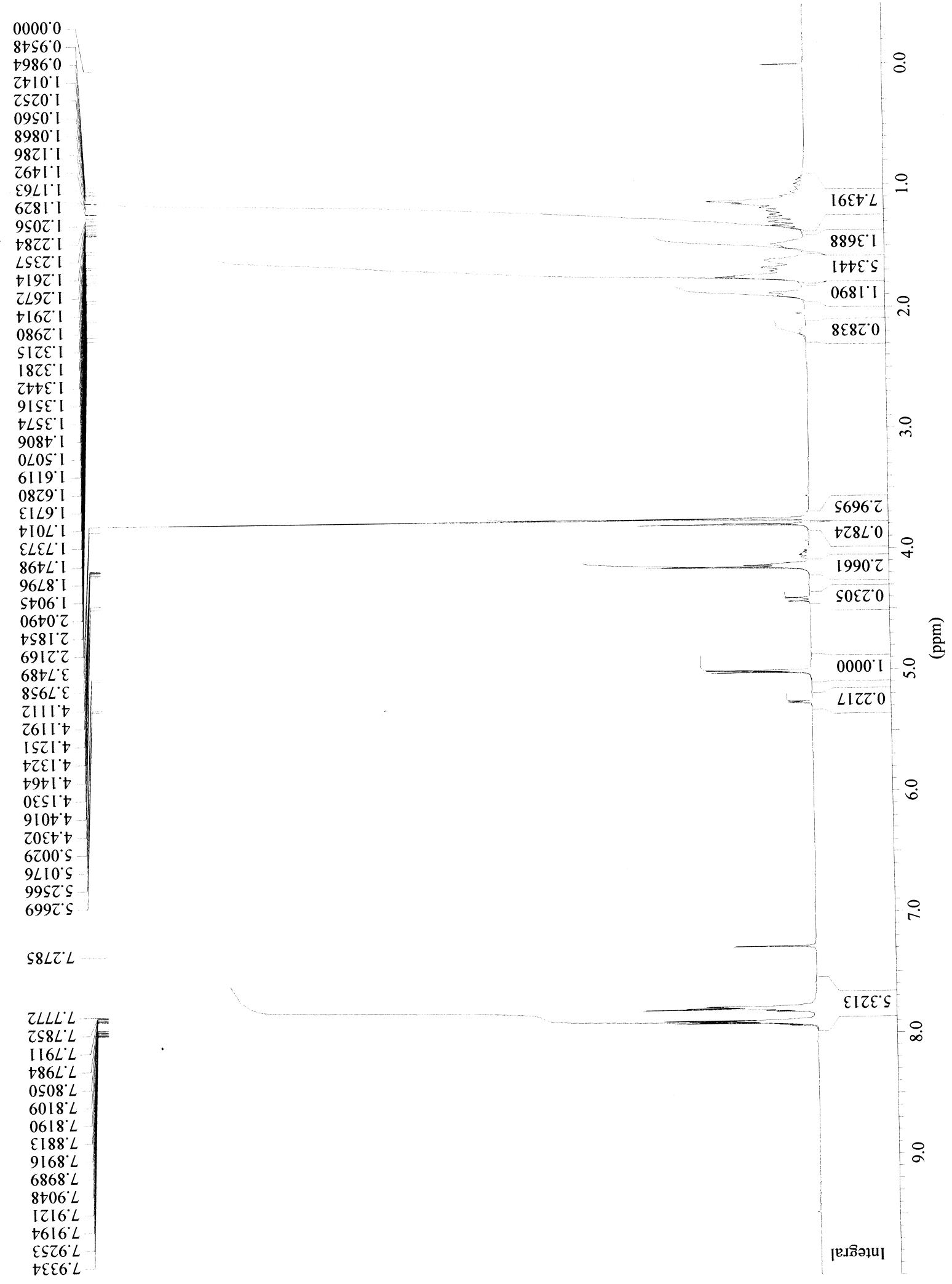


Compound 3d, anti:syn $=5: 1,{ }^{13} \mathrm{C}$ NMR $\left(125 \mathrm{MHz}, \mathrm{CDCl}_{3}\right)$

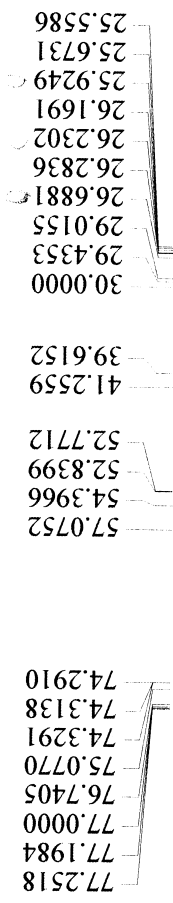

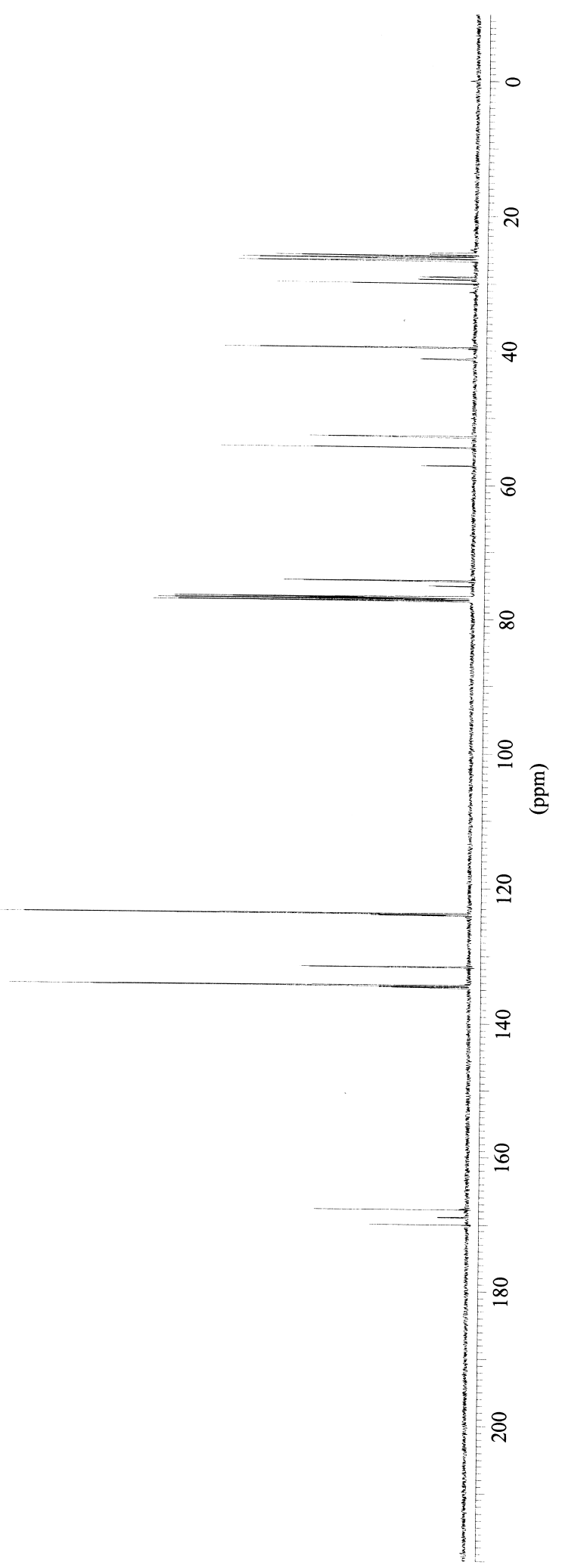


Compound 3e, ${ }^{1} \mathrm{H}$ NMR (400 MHz, $\mathrm{CDCl}_{3}$ )

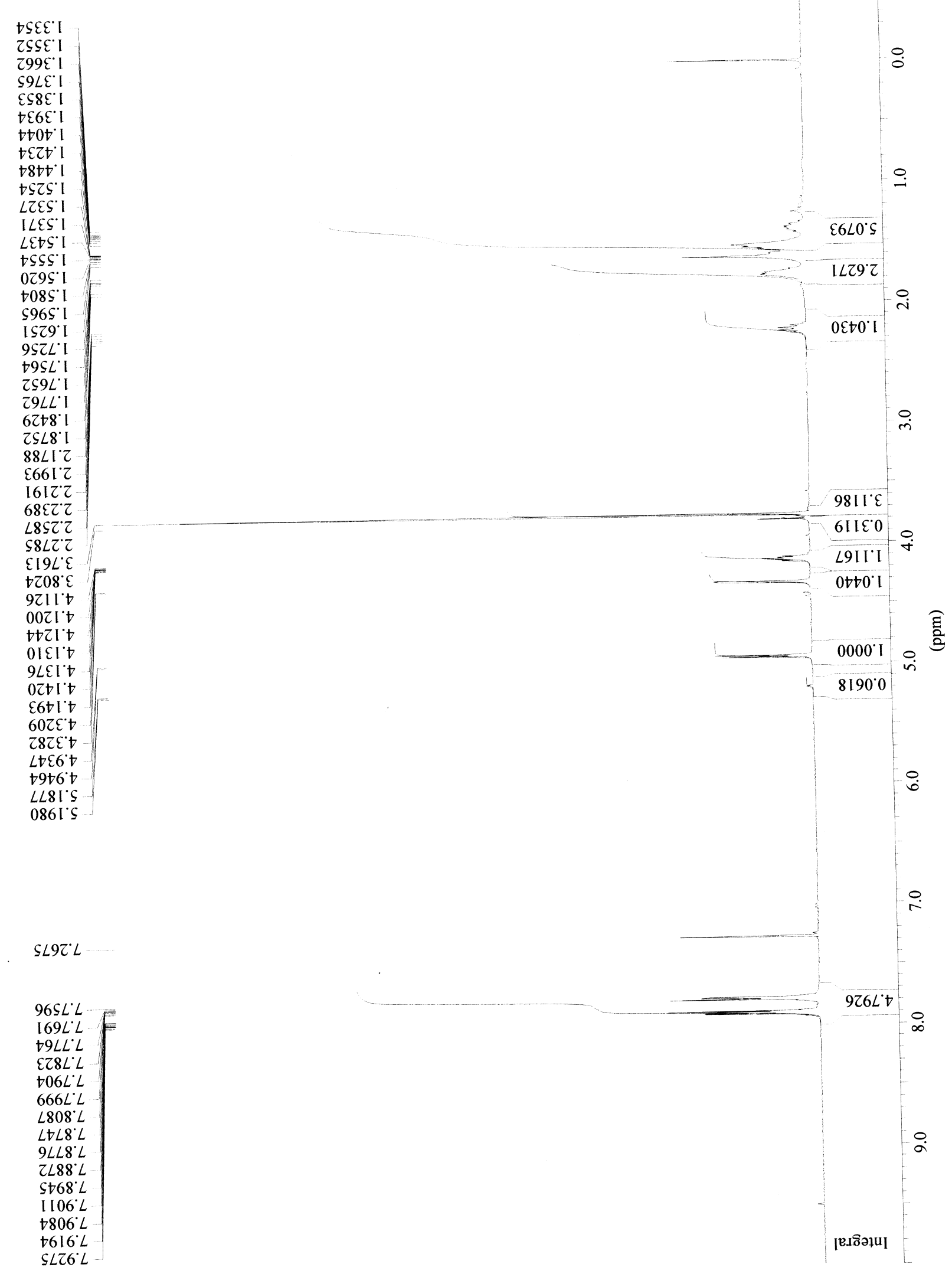




\section{Compound 3e,${ }^{13} \mathrm{C}$ NMR $\left(100 \mathrm{MHz}, \mathrm{CDCl}_{3}\right)$}

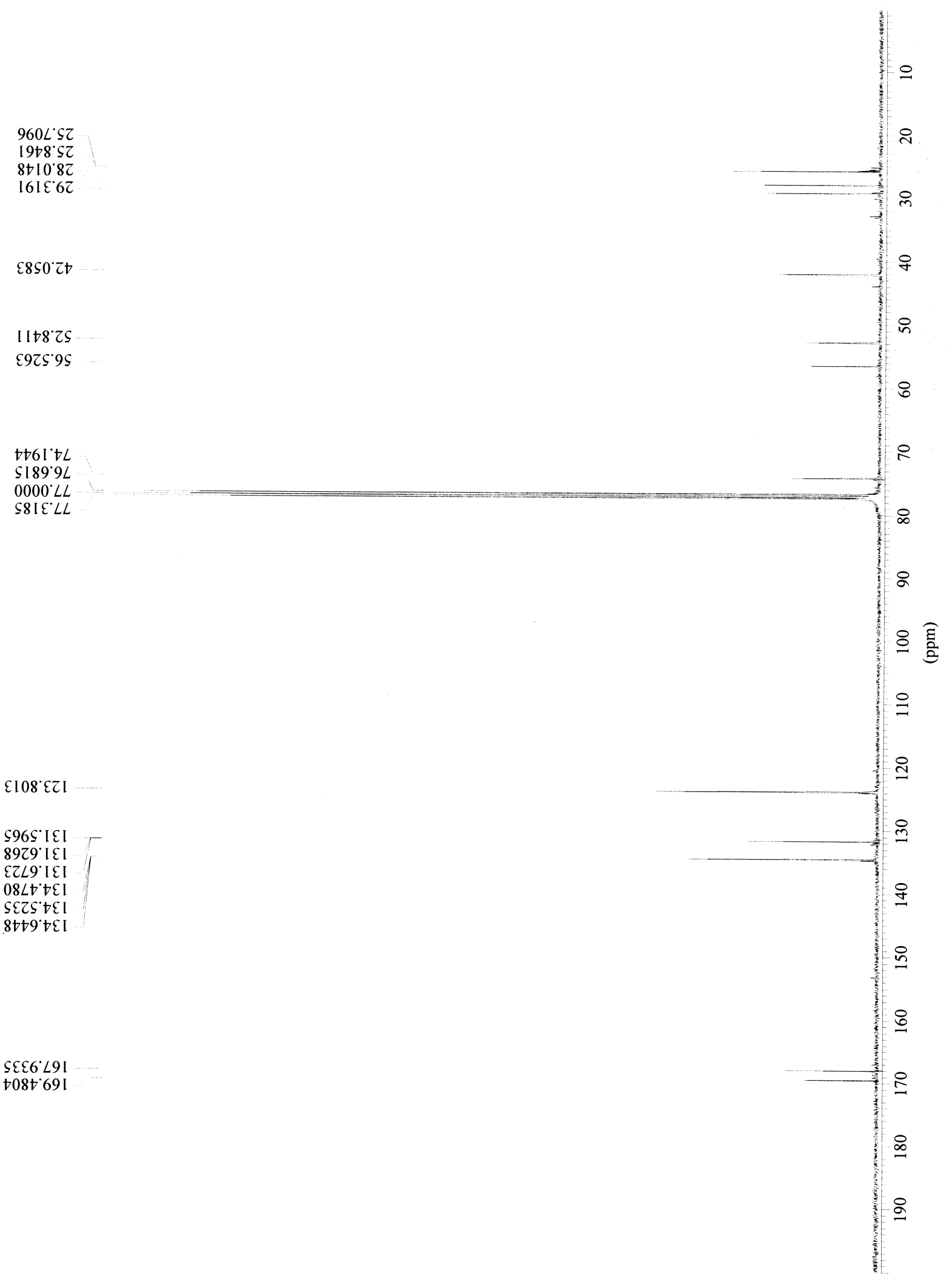


Compound 3f, anti:syn $=1: 1,{ }^{1} \mathrm{H} \mathrm{NMR}\left(400 \mathrm{MHz}, \mathrm{CDCl}_{3}\right)$

$0000^{\circ} 0$
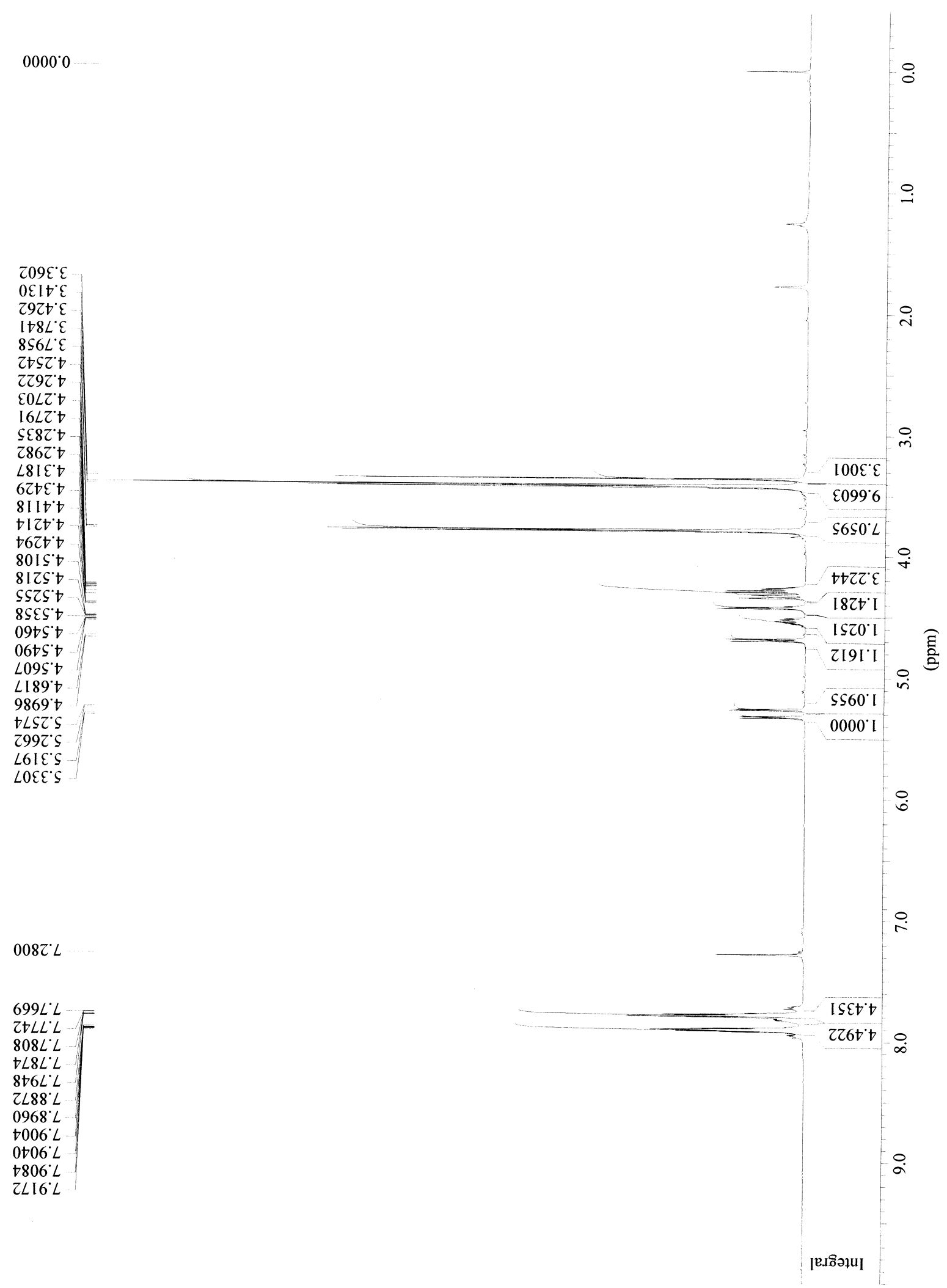

$008 Z^{\circ} L$

$699 L^{\circ} L$

$2 t L L \cdot L$

$808 L^{\circ} L$

$\checkmark \angle 8 L^{\circ} L$

$876 L^{\circ} L$

$Z \angle 88^{\circ} \angle$

$0968^{\circ} \mathrm{L}$

t006 L

$0+06^{\circ} \mathrm{L}$

$7806^{\circ} \mathrm{L}$

ZLI6 L 
Compound 3f, anti:syn $=5: 1,{ }^{13} \mathrm{C}$ NMR $\left(125 \mathrm{MHz}, \mathrm{CDCl}_{3}\right)$

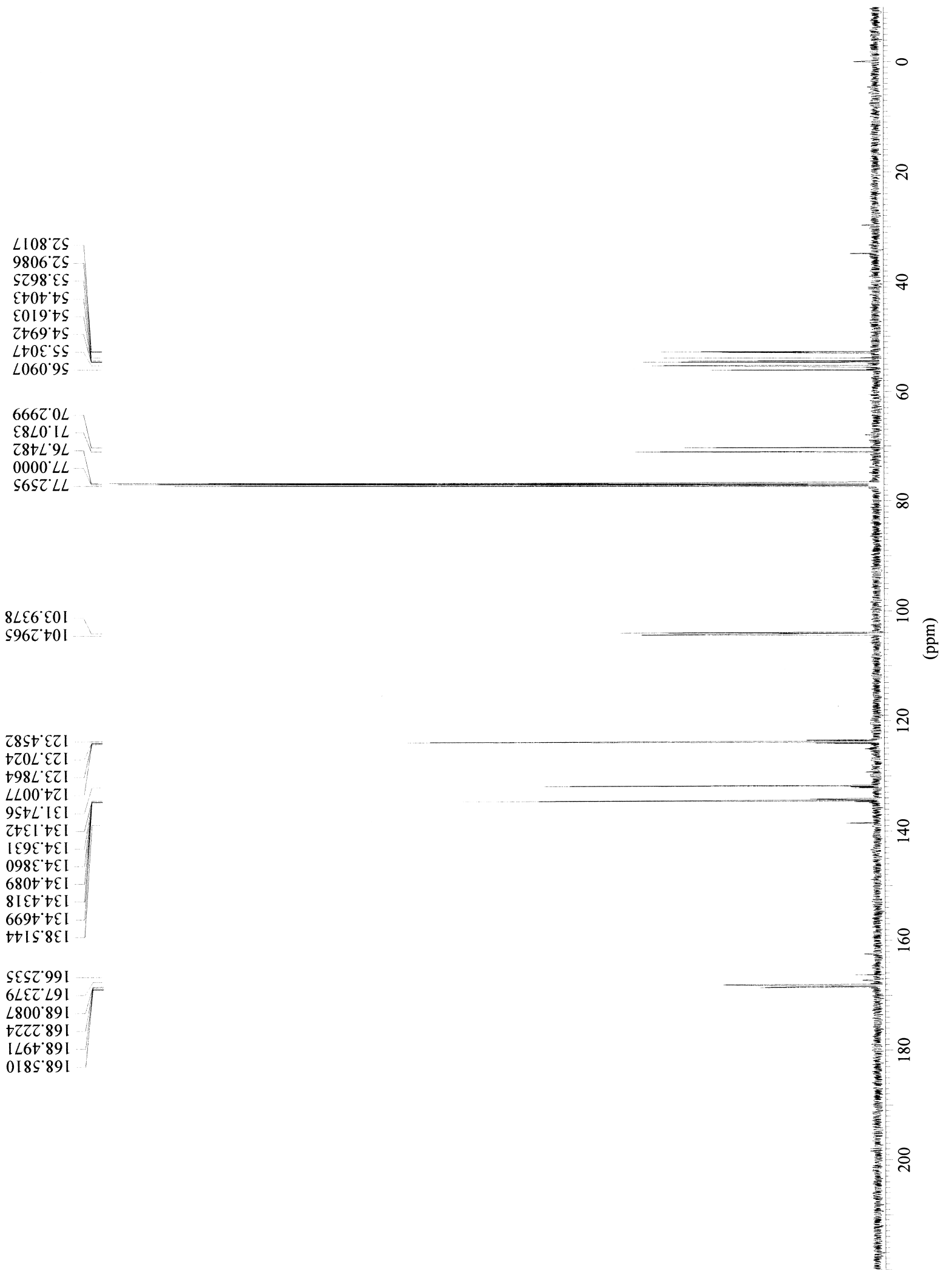


Compound6a, ${ }^{1} \mathrm{H}$ NMR $\left(400 \mathrm{MHz}, \mathrm{CDCl}_{3}\right)$

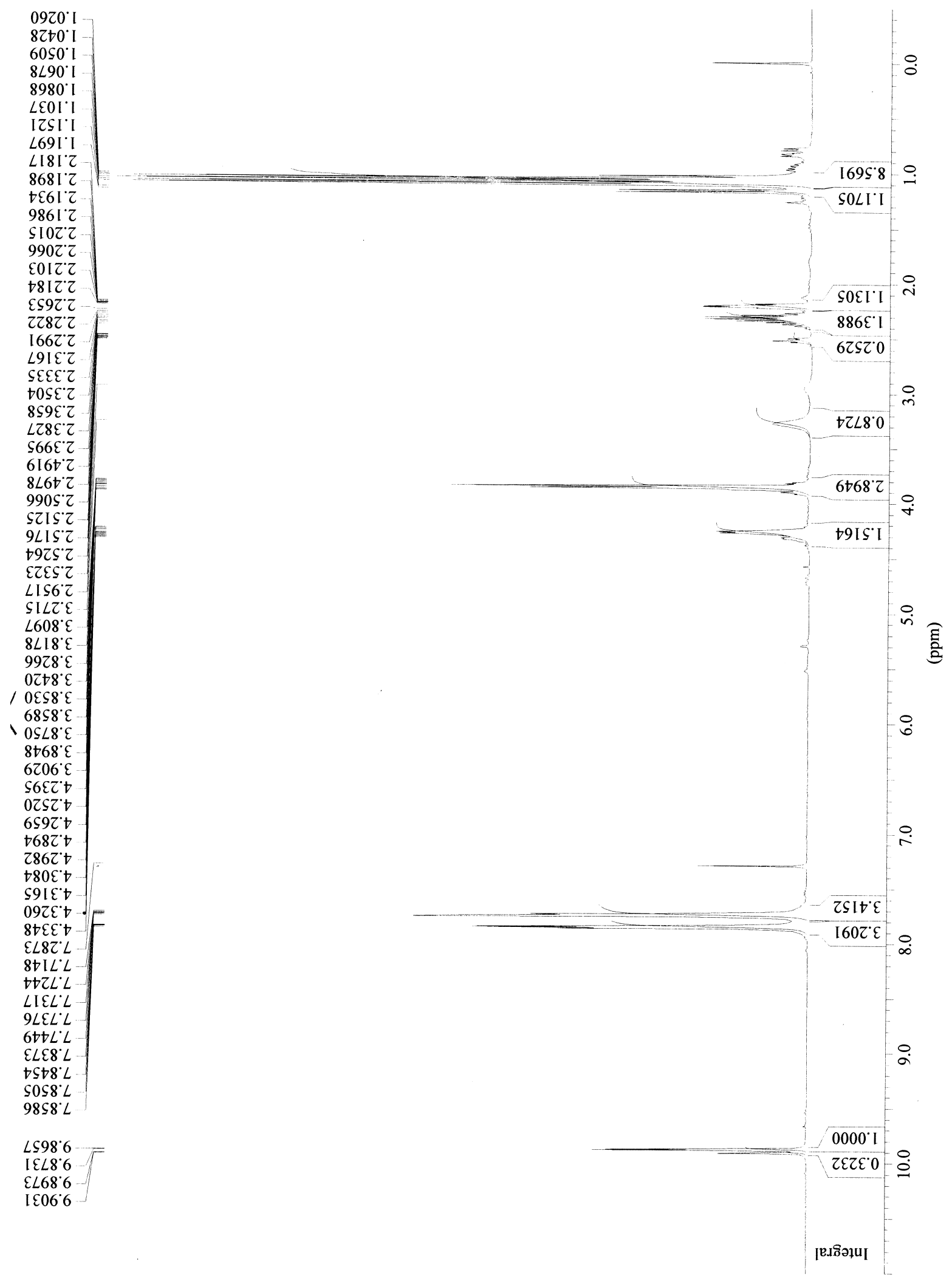


Compound 6a, ${ }^{13} \mathrm{C}$ NMR $\left(125 \mathrm{MHz}, \mathrm{CDCl}_{3}\right)$
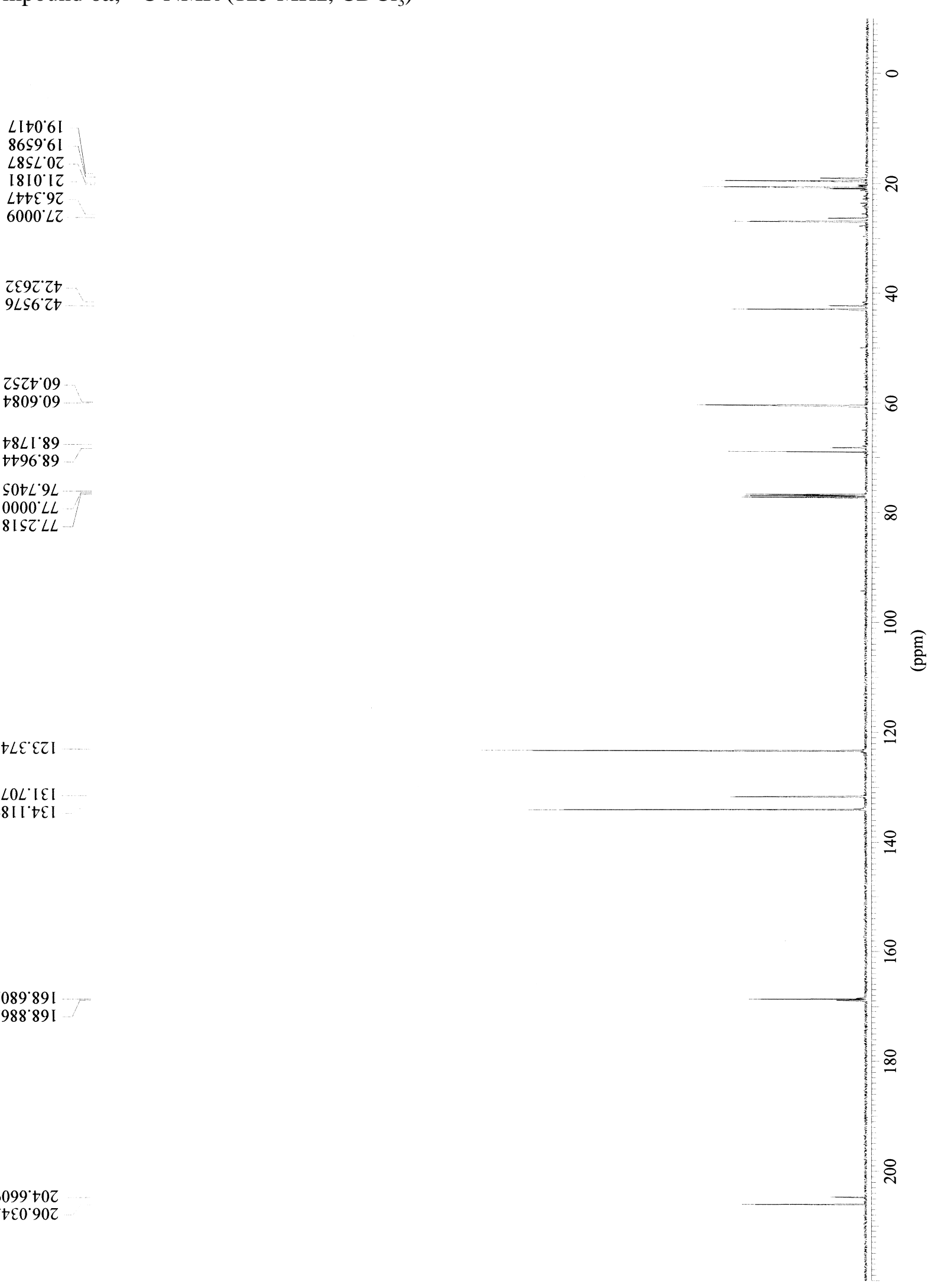